\title{
Factores predictores de sintomatología de ansiedad, depresión y calidad de vida global en pacientes mexicanos con cáncer de próstata ${ }^{1}$
}

\author{
Predictive factors for quality of life, \\ anxious, and depressive symptoms \\ in Mexican patients with prostate cancer \\ Marcos Espinoza Bello, Oscar Galindo Vázquez, \\ Miguel Angel Jiménez Ríos, Abel Lerma Talamantes, \\ Nancy Ariadna Acosta Santos, Miguel Angel Álvarez Avitia \\ y Ángel Herrera Gómez ${ }^{2}$
}

Citación: Espinoza B., M., Galindo V., Ó, Jiménez R., M.Á., Lerma T., A., Acosta S., N.A., Álvarez A., M.Á. y Herrera G., Á. (2020). Factores predictores de sintomatología de ansiedad, depresión y calidad de vida global en pacientes mexicanos con cáncer de próstata. Psicología y Salud, 30(2), 141-152. https://doi.org/10.25009/pys.v30i2.2649.

RESUMEN

\begin{abstract}
Introducción: El cáncer de próstata es el tumor más frecuente en los hombres en todo el mundo. Algunos de sus principales síntomas son fatiga, problemas urinarios, depresión y ansiedad. Por ello, conocer la calidad de vida y los problemas psicológicos en el paciente mexicano con cáncer de próstata es relevante. Objetivo: Identificar factores predictores de sintomatología de ansiedad, depresión y calidad de vida global. Método: En este estudio transversal, no experimental, participaron 201 pacientes mexicanos del Instituto Nacional de Cancerología diagnosticados con cáncer de próstata, con edades de entre 49 y 90 años. Instrumentos: Se emplearon la Escala de Ansiedad y Depresión Hospitalaria (HADS) y la Evaluación Global de Salud de la Organización Europea para la Investigación y Tratamiento del Cáncer y Calidad de Vida (EORTC QLQ-C30). Análisis estadístico: Se utilizó la prueba de chi cuadrada, la prueba exacta de Fisher y modelos de regresión logística para evaluar la fuerza de la asociación. Resultados: Los pacientes con baja calidad de vida fueron los de mayor edad, menor escolaridad, con más casos con metástasis y mayor depresión y ansiedad. Los hallazgos indican que los síntomas anteriores predicen baja calidad de vida en población mexicana. Discusión: Los síntomas clínicos de depresión y la ansiedad son predictores de baja calidad de vida en varones mexicanos, y su prevalencia en todo el espectro de tratamiento es relativamente alta. Se requieren programas de psicooncología que identifiquen y traten de forma oportuna al grupo de pacientes que lo requiera.
\end{abstract}

Palabras clave: Ansiedad; Calidad de vida; Cáncer de próstata; Depresión; Factores predictores; México.

\begin{abstract}
Background: Prostate cancer has become the most frequent tumor in men worldwide; the main physical symptoms are: fatigue 25\%, urinary problems $10 \%$ to $15 \%$, erectile dysfunction $15 \%$, psychological symptoms, depression $17 \%$ to $18 \%$, and anxiety $18 \%$ to $27 \%$. Therefore, quality of
\end{abstract}

\footnotetext{
${ }^{1}$ Los autores agradecen al Consejo Nacional de Ciencia y Tecnología (CONACyT) por el apoyo otorgado a esta investigación mediante la beca 697234.

${ }^{2}$ Instituto Nacional de Cancerología, Calle Av. San Fernando No.22, Colonia. Sección Xvi, 14080 Ciudad de México, México, correos electrónicos: wolfx17@msn.com,psigalindo@yahoo.com.mx, incanurologia@gmail.com, aleta_44@yahoo.ca, aryacos@hotmail.com y aherreragc@incan.edu.mx. Artículo recibido el 14 de agosto y aceptado el 14 de noviembre de 2019.
} 
life in Mexican patients with prostate cancer has become an important variable to measure. Objective: To identify the prevalence of anxiety and depressive symptoms, global quality of life as well as psychosocial, clinical and sociodemographic predictors of quality of life. Participants: A total of 201 patients from Mexico's National Institute of Cancerology diagnosed with prostate cancer aged 49 to 90 years. Measurement: Hospital Anxiety and Depression Scale (HADS) and Scale of the European Organization for Cancer Research and Treatment and Quality of Life (EORTC QLQ-C30) Statistical analyses: Chi square or Fisher's exact test, analysis of logistic regression models to evaluate strength of association and explanatory and predictive power. Results: Patients with poor quality of life were older, less schooled, showed more cases with metastasis and greater depressive and anxiety symptoms. Discussion: The findings indicate that the variables of depression and anxiety are predictors of low quality of life in this type of Mexican population.

Keywords: Quality of life; Prostate cancer; Anxiety; Depression; Predictor factor; Mexico.

\section{INTRODUCCIÓN}

$\mathrm{L}$ a incidencia mundial estimada del cáncer de próstata es de 1,276,106 casos, con una mortalidad de 4.06/100,000 habitantes y una prevalencia a cinco años de $3,724,658$ casos, equivalentes a 96.73/100,000 habitantes (Bray et al., 2018). En los países desarrollados hay una incidencia de 758,700 casos estimados de cáncer de próstata; esto es, de 70/100,000 habitantes, y una mortalidad de 10/100,000 habitantes; a su vez, en los países menos desarrollados hay incidencia estimada de 353,000 casos, $15 / 100,000$ habitantes y mortalidad de 165,500, es decir, 7/100,000 habitantes (Torre et al., 2015).

En México, en 2018 se registraron 25,049 casos, equivalente a 14.60/100.000 habitantes, ubicándose así en el primer tipo de neoplasia más frecuente en la población masculina. Hasta ese año, la cifra de muertes por dicho cáncer fue de 6,915 (8.99/100,000 habitantes). Así, según las estadísticas, es el tipo de cáncer que ha provocado el mayor número de muertes, incluso más que el de mama (Bray et al., 2018).
En los últimos trece años la mortalidad por cáncer de próstata en México mostró un incremento constante ( $2 \%$ anual), principalmente en los estados de la República Mexicana de muy alta (4.4\%) y alta marginación (7.7\%), mientras que en los de marginación baja hubo una reducción de 5\% anual (Torres et al., 2016).

En relación a la salud mental, se ha identificado que la depresión se presenta en $17 \%$ de este grupo de pacientes durante el pretratamiento, en $15 \%$ en el tratamiento, y en $18 \%$ en el postratamiento (Watts et al., 2014). La evidencia actualmente disponible sugiere que la privación de andrógenos en el tratamiento se asocia con un mayor riesgo de depresión (Nead, Sinha, Yang y Nguyen, 2017).

De forma similar, la ansiedad se manifiesta en $27 \%$ en el pretratamiento, en $15 \%$ en el tratamiento y en $18 \%$ en el postratamiento (Watts et al., 2014). En pacientes con enfermedad avanzada o con castración quirúrgica el riesgo de incidencia para sufrir una enfermedad psiquiátrica es de hasta $26 \%$, y entre tales problemas se hallan la depresión, la demencia, la ansiedad, el insomnio y la psicosis (Bourke et al., 2015).

Se tiene evidencia de que $16 \%$ de los pacientes muestran niveles de angustia psicológica, y $6 \%$ de síntomas de problemas mentales graves. La falta de apoyo positivo y la amenaza percibida de cáncer son predictores de comorbilidad psicológica; así como la ausencia de apoyo positivo hace que los tratamientos del cáncer anuncien una pobre salud física (Mehnert, Lehmann, Graefen, Huland y Koch, 2010).

En supervivientes de cáncer de próstata, Dale, Bilir, Han y Meltzer (2005) señalan que la ansiedad aparece en 10-36\%. En otro estudio, Jayadevappa, Malkowicz, Chhatre, Johnson y Gallo (2012) reportan que $8.5 \%$ de los pacientes manifestaban depresión durante el diagnóstico, lo que se ha asociado a las visitas a los servicios de urgencias médicas, hospitalizaciones y riesgo de morir.

En este contexto, la calidad de vida de este grupo de pacientes es una variable estrechamente relacionada con síntomas psicológicos tales como la ansiedad y depresión. Cella (1995) define la calidad de vida como "el grado en que se ven 
afectados el bienestar habitual físico, emocional y social [de los pacientes] debido a una condición médica o su tratamiento". La Organización Mundial de la Salud (OMS) (cf. The WHOQOL Group, 1998) la define a su vez como "la percepción que un individuo tiene de su lugar en la existencia, en el contexto de la cultura y del sistema de valores en los que vive en relación con sus objetivos, sus expectativas, sus normas, sus inquietudes". En el campo de la oncología, dicha definición proporciona los requerimientos mínimos en razón de que la perspectiva del paciente se obtiene en las áreas física y mental y en el bienestar social tomando en cuenta la multidimensionalidad del constructo (Wenzel y Cella, 2005).

Los principales síntomas físicos de este grupo de pacientes son, a saber: astenia (25\%) (Monga, Kerrigan, Thornby, Monga y Zimmermann, 2005); flujo de orina débil o flujo interrumpido (10-15\%), aumento en la frecuencia de micciones a dos años de una prostatectomía (50-60\%), diarreas debidas a una braquiterapia (15-40\%) y disfunción eréctil (15\%), que en pacientes con radioterapia ocurre entre 30 y 40\%; además, $70 \%$ de los pacientes con cáncer de próstata sufre dolor de cadera, espalda o tórax debido a la propagación del cáncer en huesos u otras áreas (American Cancer Society, 2016; Chen et al., 2014).

Oraá et al. (2017) observaron las diferencias en la calidad de vida y la información en 176 pacientes con cáncer de próstata seleccionados aleatoriamente, quienes recibieron tratamientos locales, hormonales o combinados. Los pacientes tratados con radioterapia pensaban que entendían mejor la información, en comparación con los aquellos a quienes se practicó la prostatectomía, y además que esta última producía problemas urinarios más graves y que los tratamientos combinados generaban una mayor sintomatología hormonal.

En México hay escasa investigación respecto a calidad de vida y la salud mental en este grupo de pacientes. Solamente se pudo identificar el estudio de Sierra, Viveros, Martínez, Hernández y Caballero (2014), quienes reportan un impacto negativo en la calidad de vida, síntomas urinarios y efectos secundarios de terapias adyuvantes, como la hormonoterapia y radioterapia, en 32 pacientes operados, y la investigación de Vázquez, Camarena, Martínez y Hernández (2009), quienes encontraron afectaciones en la calidad de vida respecto al funcionamiento urinario, sexual e intestinal en 57 pacientes que fueron sujetos a prostatectomía radical, más adyuvancia con radioterapia y bloqueo hormonal.

El presente estudio tuvo como objetivos identificar los factores predictores de calidad de vida global, sintomatología de ansiedad y depresión; así como comparar las características sociodemográficas y clínicas entre grupos con mayor calidad de vida global y sintomatología psicológica.

\section{MÉTODO}

\section{Participantes}

Participaron 201 pacientes de la Unidad Funcional de Urología del Instituto Nacional de Cancerología (INCan), ubicado en la Ciudad de México. Previa aprobación del estudio por el Comité de Ética e Investigación de dicha institución, se efectuó un muestreo no probabilístico y por disponibilidad. El estudio fue transversal, no experimental, y el tamaño de la muestra de participantes se calculó considerando el criterio de Nunally y Bernstein (1995), mediante el cual se multiplica el número total de reactivos por 5 (participantes por cada reactivo), lo que da un total de 215 . Únicamente se incluyeron en el análisis aquellos pacientes que cubrían los criterios que se mencionan a continuación: tener diagnóstico de cáncer de próstata; estar en tratamiento oncológico de cualquier tipo y en cualquier estadio, o en periodo de sobrevida, y saber leer y escribir. Los criterios de exclusión fueron padecer deficiencias cognitivas, auditivas o visuales. Se eliminaron aquellos que decidieron no seguir participando o que no completaron el llenado de los instrumentos.

Mediante una entrevista directa con el paciente y a través de la consulta de su expediente clínico se obtuvo la información necesaria sobre variables, tales como edad, escolaridad, estado civil, nivel económico, ocupación, número de hijos, etapa clínica, tratamientos y número de comorbi- 
lidades. Los datos de las variables psicológicas (calidad de vida global, síntomas de depresión y ansiedad) se midieron empleando los instrumentos que se describen a continuación.

\section{Instrumentos}

Escala de Ansiedad y Depresión Hospitalaria (HADS) (Zigmond y Snaith, 1983).

Ya validada en México por Galindo et al. (2015), mide sintomatología ansiosa y depresiva a través de doce reactivos. En su versión mexicana posee una consistencia interna global y por subescalas (coeficientes alfa de 0.86 en la escala total y en cada subescala coeficientes que van de 0.79 a 0.80 ), que explican en conjunto $48.04 \%$ de la varianza. Según los puntos de corte para la versión mexicana, se considera la presencia de sintomatología depresiva o ansiosa a partir del punto 6 .

\section{Escala de la Organización Europea}

para la Investigación y Tratamiento del Cáncer

y Calidad de Vida (QLQ-C30) (Aaronson et al., 1993).

Validada en México por Oñate et al., (2009), es un instrumento que mide calidad de vida y es específico para cáncer y calidad de vida global; posee un coeficiente alfa entre dimensiones que va de 0.60 a 0.70. En este caso, se empleó la subescala de Calidad de Vida Global, que consta de 30 reactivos.

\section{Análisis estadísticos}

Las variables nominales $\mathrm{u}$ ordinales se describieron mediante frecuencias y porcentajes; para las comparaciones se utilizó la chi cuadrada o la exacta de Fisher, y para variables ordinales y continuas se encontró que su distribución no era normal (prueba de Kolmogorov-Smirnov), por lo que se describieron mediante la mediana (percentiles 25-75) Se hicieron comparaciones empleando la prueba U de Mann Whitney por grupos respecto a la calidad de vida (definida como menor o mayor usando como punto de corte el valor de la mediana de la puntuación total), presencia de sintomatología depresiva (puntuación total de sintomatología de depresión $\geq 6$ puntos) y presencia de sintomatología ansiosa (puntuación total de sintomatología de ansiedad $\geq 6$ puntos) usando para ello los puntos de corte señalados por Galindo et al (2015). Con las variables que mostraban una diferencia significativa entre los grupos en cada uno de estos desenlaces, se evaluó la asociación de las variables con cada uno de ellos mediante un análisis de regresión logística multivariada de los factores asociados con mala calidad de vida y la presencia de sintomatología depresiva o ansiosa. Se reportan los resultados como razón de momios e intervalo de confianza a 95\%. Las correlaciones bivariadas entre las puntuaciones totales de calidad de vida, depresión y ansiedad se evaluaron mediante el coeficiente de Spearman. Se consideró significativo un valor $p \leq 0.05$. El análisis estadístico se efectuó utilizando el programa SPSS, versión 15.0 (Statsoft, Inc.).

\section{RESULTADOS}

La Tabla 1 muestra las características sociodemográficas, clínicas y psicológicas comparadas respecto a la calidad de vida global. En el análisis comparativo, los pacientes con menor calidad de vida tuvieron mayor edad, menor escolaridad, más casos con metástasis y más síntomas de depresión y ansiedad que los pacientes con mayor calidad de vida.

En el análisis comparativo entre los pacientes con y sin síntomas clínicos de depresión, hubo más pacientes que vivían solos o sin pareja, que no tenían un tratamiento para el cáncer o que solo estaban sometidos a uno, con calidad de vida menor, mayores puntajes totales de depresión y ansiedad, y más casos con presencia de ansiedad comparados con los que no manifestaban síntomas de depresión (Tabla 2). 
Tabla 1. Características socio demográficas, clínicas y psicológicas comparadas respecto a la calidad de vida global. Los valores se muestran en frecuencias y porcentajes o en medianas (percentil 25 - percentil 75).

\begin{tabular}{|c|c|c|c|}
\hline \multirow[b]{2}{*}{ Variable (Poder estadístico) } & \multicolumn{2}{|c|}{ Calidad de vida global } & \multirow[b]{2}{*}{$p$} \\
\hline & $\begin{array}{c}\text { Menor } \\
<75 \text { puntos } \\
(\mathrm{N}=99)\end{array}$ & $\begin{array}{c}\text { Mayor } \\
\geq 75 \text { puntos } \\
(\mathrm{N}=102)\end{array}$ & \\
\hline Edad (años) & $70(64-74)$ & $67(63-71)$ & 0.027 \\
\hline \multicolumn{3}{|l|}{ Escolaridad } & 0.001 \\
\hline Ninguna o básica & $77(78)$ & $56(55)$ & \\
\hline Media a superior & $22(22)$ & $46(45)$ & \\
\hline \multicolumn{3}{|l|}{ Estado civil } & 0.388 \\
\hline En pareja & $26(26)$ & $24(24)$ & \\
\hline Sin pareja & $73(74)$ & $78(77)$ & \\
\hline \multicolumn{3}{|l|}{ Nivel económico } & 0.072 \\
\hline Bajo a Medio & $94(95)$ & $90(88)$ & \\
\hline Alto & $5(5)$ & $12(12)$ & \\
\hline \multicolumn{3}{|l|}{ Ocupación } & 0.071 \\
\hline Inactivo económicamente & $45(45)$ & $35(34)$ & \\
\hline Activo económicamente & $54(55)$ & $67(66)$ & \\
\hline \multicolumn{3}{|l|}{ Número de hijos } & 0.332 \\
\hline Dos o menos & $33(33)$ & $38(37)$ & \\
\hline Tres o más & $66(67)$ & $64(63)$ & \\
\hline \multicolumn{3}{|l|}{ Etapa clínica } & 0.002 \\
\hline Metástasis & $44(44)$ & $24(24)$ & \\
\hline Sin metástasis & $55(56)$ & $78(76)$ & \\
\hline \multicolumn{3}{|l|}{ Tratamientos } & 0.358 \\
\hline Sin tratamiento o hasta uno & $54(55)$ & $52(51)$ & \\
\hline Dos o más tratamientos & $45(45)$ & $50(49)$ & \\
\hline \multicolumn{3}{|l|}{ Número comorbilidades } & 0.284 \\
\hline Ninguna & $53(54)$ & $44(43)$ & \\
\hline Una comorbilidad & $40(40)$ & $48(47)$ & \\
\hline Dos o más comorbilidades & $6(6)$ & $10(10)$ & \\
\hline \multicolumn{3}{|l|}{ Sintomatología de depresión } & \\
\hline Puntuación total & $7(4-10)$ & $2(1-5)$ & $<0.001$ \\
\hline Presencia de depresión & & & $<0.001$ \\
\hline No (puntuación < 6) & $52(53)$ & $92(90)$ & \\
\hline Sí (puntuación $\geq 6$ ) & $47(47)$ & $10(10)$ & \\
\hline \multicolumn{3}{|l|}{ Sintomatología de ansiedad } & \\
\hline Puntuación total & $3(1-6)$ & $1(1-3)$ & $<0.001$ \\
\hline Presencia de ansiedad & & & $<0.001$ \\
\hline No (puntuación <6) & $73(74)$ & $99(97)$ & \\
\hline Sí (puntuación $\geq 6$ ) & $26(26)$ & $3(3)$ & \\
\hline
\end{tabular}


Tabla 2. Características sociodemográficas, clínicas y psicológicas comparadas respecto a depresión. Los valores se muestran en frecuencias y porcentajes o en medianas (percentil 25 - percentil 75).

\begin{tabular}{|c|c|c|c|}
\hline \multicolumn{4}{|c|}{ Sintomatología depresiva } \\
\hline Variables del estudio & $\begin{array}{l}\text { Síntomas clínicos } \\
\text { de depresión } \\
\geq 6 \text { puntos } \\
(\mathrm{N}=27)\end{array}$ & $\begin{array}{l}\text { Sin depresión } \\
<6 \text { puntos } \\
(\mathrm{N}=174)\end{array}$ & $p$ \\
\hline Edad (años) & $68(64-71)$ & $68(63-73)$ & 0.859 \\
\hline \multicolumn{3}{|l|}{ Escolaridad } & \multirow{3}{*}{0.171} \\
\hline Ninguna o Básica & $21(78)$ & $112(64)$ & \\
\hline Media a Superior & $6(22)$ & $62(36)$ & \\
\hline \multicolumn{3}{|l|}{ Estado civil } & \multirow{3}{*}{0.003} \\
\hline En pareja & $1(4)$ & $49(28)$ & \\
\hline Sin pareja & $26(96)$ & $125(72)$ & \\
\hline \multicolumn{3}{|l|}{ Nivel económico } & \multirow{3}{*}{0.077} \\
\hline Bajo a Medio & $27(100)$ & $157(90)$ & \\
\hline Alto & $0(0)$ & $17(10)$ & \\
\hline \multicolumn{3}{|l|}{ Ocupación } & \multirow{3}{*}{0.915} \\
\hline Inactivo económicamente & $11(41)$ & $69(40)$ & \\
\hline Activo económicamente & $16(59)$ & $105(60)$ & \\
\hline \multicolumn{3}{|l|}{ Número de hijos } & \multirow{3}{*}{0.500} \\
\hline Dos o menos & $9(33)$ & $62(36)$ & \\
\hline Tres o más & $18(67)$ & $112(64)$ & \\
\hline \multicolumn{3}{|l|}{ Etapa clínica } & \multirow{3}{*}{0.091} \\
\hline Metástasis & $14(52)$ & $119(68)$ & \\
\hline Sin metástasis & $13(48)$ & $55(32)$ & \\
\hline \multicolumn{3}{|l|}{ Tratamientos } & \multirow{3}{*}{0.014} \\
\hline Sin tratamiento o hasta uno & $20(74)$ & $86(49)$ & \\
\hline Dos o más tratamientos & $7(26)$ & $88(51)$ & \\
\hline \multicolumn{3}{|l|}{ Número comorbilidades } & \multirow{4}{*}{0.923} \\
\hline Ninguna & $14(52)$ & $83(47)$ & \\
\hline Una comorbilidad & $11(41)$ & $77(44)$ & \\
\hline Dos o más comorbilidades & $2(7)$ & $14(8)$ & \\
\hline \multicolumn{3}{|l|}{ Calidad de vida global } & \multirow{3}{*}{$<0.001$} \\
\hline Menor $<75$ puntos) & $23(85)$ & $76(44)$ & \\
\hline Mayor $\geq 75$ puntos) & $4(15)$ & $98(56)$ & \\
\hline \multicolumn{3}{|l|}{ Sintomatología de ansiedad } & \multirow{2}{*}{$<0.001$} \\
\hline Puntuación total & $6(4-8)$ & $2(1-3)$ & \\
\hline \multicolumn{3}{|c|}{ Presencia de sintomatología de ansiedad } & \multirow{3}{*}{$<0.001$} \\
\hline No (puntuación <6) & $13(48)$ & $159(91)$ & \\
\hline Sí (puntuación $\geq 6$ ) & $14(52)$ & $15(9)$ & \\
\hline
\end{tabular}

La comparación de los síntomas clínicos de ansiedad indica que los pacientes ansiosos eran más jóvenes, tenían menor calidad de vida global, mayor puntaje de depresión total y más casos con presen- cia de depresión, comparados con los no ansiosos (Tabla 3).

El análisis de correlación bivariada de Spearman en las puntuaciones totales de las variables 
Tabla 3. Características sociodemográficas, clínicas y psicológicas comparadas respecto a la ansiedad. Los valores se muestran en frecuencias y porcentajes o en medianas (percentiles 25-75).

\begin{tabular}{|c|c|c|c|}
\hline \multirow[b]{2}{*}{ Variables del estudio } & \multicolumn{2}{|c|}{ Sintomatología de ansiedad } & \multirow[b]{2}{*}{$p$} \\
\hline & $\begin{array}{l}\text { Síntomas clínicos } \\
\text { de ansiedad } \\
\geq 6 \text { puntos } \\
(\mathrm{N}=29)\end{array}$ & $\begin{array}{c}\text { Sin ansiedad } \\
<6 \text { puntos } \\
(\mathrm{N}=172)\end{array}$ & \\
\hline Edad (años) & $67(61-70)$ & $69(64-74)$ & 0.035 \\
\hline \multicolumn{3}{|l|}{ Escolaridad } & \multirow{3}{*}{0.614} \\
\hline Ninguna o básica & $18(62)$ & $115(67)$ & \\
\hline Media a superior & $11(38)$ & $57(33)$ & \\
\hline \multicolumn{3}{|l|}{ Estado civil } & \multirow{3}{*}{0.573} \\
\hline Sin pareja & $6(21)$ & $44(26)$ & \\
\hline Con pareja & $23(79)$ & $128(74)$ & \\
\hline \multicolumn{3}{|l|}{ Nivel económico } & \multirow{3}{*}{0.063} \\
\hline Bajo a medio & $29(100)$ & $155(90)$ & \\
\hline Alto & $0(0)$ & $17(10)$ & \\
\hline \multicolumn{3}{|l|}{ Ocupación } & \multirow{3}{*}{0.053} \\
\hline Inactivo económicamente & $16(55)$ & $65(37)$ & \\
\hline Activo económicamente & $13(45)$ & $108(63)$ & \\
\hline \multicolumn{3}{|l|}{ Número de hijos } & \multirow{3}{*}{0.461} \\
\hline Dos o menos & $12(41)$ & $59(34)$ & \\
\hline Tres o más & $17(59)$ & $113(66)$ & \\
\hline \multicolumn{3}{|l|}{ Etapa clínica } & \multirow{3}{*}{0.614} \\
\hline Con metástasis & $18(62)$ & $115(67)$ & \\
\hline Sin metástasis & $11(38)$ & $57(33)$ & \\
\hline \multicolumn{3}{|l|}{ Tratamientos } & \multirow{3}{*}{0.906} \\
\hline Sin tratamiento o hasta uno & $15(52)$ & $91(53)$ & \\
\hline Dos o más tratamientos & $14(48)$ & $81(47)$ & \\
\hline \multicolumn{3}{|l|}{ Número comorbilidades } & \multirow{4}{*}{0.219} \\
\hline Ninguna & $16(55)$ & $81(47)$ & \\
\hline Una comorbilidad & $9(31)$ & $79(46)$ & \\
\hline Dos o más comorbilidades & $4(14)$ & $12(7)$ & \\
\hline \multicolumn{3}{|l|}{ Calidad de vida global } & \multirow{3}{*}{$<0.001$} \\
\hline Menor ( $<75$ puntos) & $26(90)$ & $73(42)$ & \\
\hline Mayor ( $\geq 75$ puntos) & $3(10)$ & $99(58)$ & \\
\hline \multicolumn{3}{|l|}{ Sintomatología depresiva } & \multirow{4}{*}{$<0.001$} \\
\hline Puntuación total & $5(4-7)$ & $1(0-4)$ & \\
\hline No (puntuación <6) & $15(52)$ & $159(92)$ & \\
\hline Sí (puntuación $\geq 6$ ) & $14(48)$ & $13(8)$ & \\
\hline
\end{tabular}

psicológicas mostró una asociación negativa entre calidad de vida y síntomas depresión $(R h o=-0.488)$ y entre calidad de vida y síntomas de ansiedad $(R h o=-0.340)$, y asociación positiva entre síntomas ansiedad y síntomas de depresión $(R h o=0.540)$, donde todas las correlaciones fueron significativas con valor $p<0.001$.

Considerando esas asociaciones, se llevaron a cabo análisis de regresión logística múltiple para identificar la fuerza de asociación de los factores 
asociados con una menor calidad de vida global y presencia de síntomas clínicos de depresión o de ansiedad mediante varios modelos que solo consideraran como variable independiente una de tipo psicológico por modelo, además de las variables sociodemográficas y clínicas que tuvieron diferencia significativa en las comparaciones por grupos. Es importante señalar que en cada variable predictora o explicativa (mala calidad de vida, depresión, ansiedad), y para eliminar el efecto de colinealidad se generaron los seis distintos modelos que se muestran en la Tabla 4.

Tabla 4. Análisis de regresión logística múltiple de los factores asociados con baja calidad de vida ( $\geq 75$ puntos) y con la presencia de depresión o síntomas de ansiedad ( $\geq 6$ puntos) en una muestra de 201 pacientes con cáncer de próstata.

\begin{tabular}{|c|c|c|c|c|}
\hline Variables & O.R. (I.C.95\%) & $p$ & O.R. (I.C.95\%) & $p$ \\
\hline & \multicolumn{2}{|l|}{ Modelo 1} & \multicolumn{2}{|l|}{ Modelo 2} \\
\hline $\begin{array}{l}\text { Modelos para menor calidad } \\
\text { de vida } \\
(\leq 75 \text { puntos })\end{array}$ & \multicolumn{2}{|c|}{$\begin{array}{l}\text { BAJA CALIDAD DE VIDA }=\text { Edad + Escolaridad } \\
+ \text { Etapa clínica }+ \text { Presencia de depresión } \\
\text { Constante }=0.286, \text { Nagelkerke } \mathrm{R}^{2}=0.213 \\
\text { Sensibilidad }=52 \% \text {, Especificidad }=80 \% \text {, } \\
\text { Porcentaje total }=66 \%\end{array}$} & \multicolumn{2}{|c|}{$\begin{array}{l}\text { BAJA CALIDAD DE VIDA }=\text { Edad + Escolaridad } \\
+ \text { Etapa clínica + Presencia de ansiedad } \\
\text { Constante }=0.096, \text { Nagelkerke } \mathrm{R}^{2}=0.298 \\
\text { Sensibilidad }=61 \%, \text { Especificidad }=80 \%, \\
\text { Porcentaje total }=71 \%\end{array}$} \\
\hline Edad (años) & $1.02(0.98-1.06)$ & 0.456 & $1.03(0.99-1.07)$ & 0.153 \\
\hline $\begin{array}{l}\text { Escolaridad } \\
\text { (ninguna o básica) }\end{array}$ & $0.40(0.21-0.78)$ & 0.007 & $0.31(0.15-0.63)$ & 0.001 \\
\hline Metástasis & $2.28(1.20-4.36)$ & 0.012 & $2.57(1.32-5.02)$ & 0.006 \\
\hline $\begin{array}{l}\text { Presencia de sintomatología } \\
\text { de depresión }\end{array}$ & $6.64(2.14-20.62)$ & 0.001 & - & - \\
\hline $\begin{array}{l}\text { Presencia de sintomatología } \\
\text { de ansiedad }\end{array}$ & - & - & $18.94(4.98-71.97)$ & $<0.001$ \\
\hline \multirow[b]{2}{*}{$\begin{array}{l}\text { Modelos para presencia } \\
\text { de sintomatología } \\
\text { de depresión ( } \geq 6 \text { puntos })\end{array}$} & \multicolumn{2}{|l|}{ Modelo 3} & \multicolumn{2}{|l|}{ Modelo 4} \\
\hline & \multicolumn{2}{|c|}{$\begin{array}{l}\text { DEPRESIÓN }=\text { Sin pareja }+ \text { Sin tratamiento } \\
\text { o uno solamente }+ \text { Mala calidad de vida } \\
\text { Constante }=0.006, \text { Nagelkerke } \mathrm{R}^{2}=0.293 \\
\text { Sensibilidad }=0 \% \text {, Especificidad }=100 \% \text {, } \\
\text { Porcentaje total }=87 \%\end{array}$} & \multicolumn{2}{|c|}{$\begin{array}{l}\text { DEPRESIÓN }=\text { Sin pareja }+ \text { Sin tratamiento } \\
\text { o uno solamente }+ \text { Presencia de ansiedad } \\
\text { Constante }=0.011, \text { Nagelkerke } \mathrm{R}^{2}=0.376 \\
\text { Sensibilidad }=33 \%, \text { Especificidad }=99 \% \text {, } \\
\text { Porcentaje total }=90 \%\end{array}$} \\
\hline Sin pareja & $12.99(1.66-101.58)$ & 0.015 & $17.63(1.95-159.75)$ & 0.011 \\
\hline $\begin{array}{l}\text { Sin tratamiento } \\
\text { o un solo tratamiento }\end{array}$ & $3.21(1.21-8.48)$ & 0.019 & $5.11(1.63-16.01)$ & 0.005 \\
\hline Baja calidad de vida & $8.44(2.72-26.20)$ & $<0.001$ & - & - \\
\hline $\begin{array}{l}\text { Presencia de sintomatología } \\
\text { de ansiedad }\end{array}$ & - & - & $18.32(5.90-56.90$ & $<0.001$ \\
\hline \multirow[b]{2}{*}{$\begin{array}{l}\text { Modelos para presencia } \\
\text { de sintomatología de ansiedad } \\
\text { ( } \geq 6 \text { puntos) }\end{array}$} & \multicolumn{2}{|l|}{ Modelo 5} & \multicolumn{2}{|l|}{ Modelo 6} \\
\hline & \multicolumn{2}{|c|}{$\begin{array}{l}\text { ANSIEDAD }=\text { Edad }+ \text { Mala calidad de vida } \\
\text { Constante }=5.01, \text { Nagelkerke } \mathrm{R}^{2}=0.271 \\
\text { Sensibilidad }=7 \%, \text { Especificidad }=98 \%, \\
\text { Porcentaje total }=85 \%\end{array}$} & \multicolumn{2}{|c|}{$\begin{array}{l}\text { ANSIEDAD }=\text { Edad }+ \text { Presencia de depresión } \\
\text { Constante }=23.51, \text { Nagelkerke } \mathrm{R}^{2}=0.274 \\
\text { Sensibilidad }=31 \%, \text { Especificidad }=97 \% \text {, } \\
\text { Porcentaje total }=87 \%\end{array}$} \\
\hline Edad & $0.93(0.88-0.98)$ & 0.006 & $0.92(0.86-0.98)$ & 0.010 \\
\hline Baja calidad de vida & $13.84(3.96-48.33)$ & $<0.001$ & - & - \\
\hline $\begin{array}{l}\text { Presencia de sintomatología } \\
\text { de depresión }\end{array}$ & - & - & $13.34(5.03-35.40$ & $<0.001$ \\
\hline
\end{tabular}

El análisis de regresión logística determinó que los factores asociados de manera independiente con baja calidad fueron poca escolaridad, metástasis, presencia de síntomas clínicos de depresión y de ansiedad (modelos 1 y 2). En el modelo 1, para la variable Mala Calidad de Vida como predictora, la variable ansiedad no se incluyó para evitar su interacción con la depresión; a la vez, en 
el modelo 2, se retiró la depresión. Considerando esta última como variable predictora, las variables asociadas independientemente fueron el hecho de vivir solo o no tener pareja, tener solamente un tratamiento o no tener ninguno, tener baja calidad de vida y padecer ansiedad (modelos 3 y 4). En estos modelos se retiraron el efecto de la baja calidad de vida o de la ansiedad, respectivamente. Por último, tomando la ansiedad como variable predictora, los factores independientemente asociados fueron tener menor edad, baja calidad de vida y depresión (modelos 5 y 6); en dichos modelos se alternaron el efecto de la baja calidad de vida o de la depresión, respectivamente.

\section{DISCUSIÓN}

El presente estudio tuvo por objetivo determinar los factores predictores psicosociales, clínicos y sociodemográficos de la sintomatología depresiva, ansiosa y calidad de vida global en pacientes con cáncer de próstata. Se identificaron factores asociados con baja calidad de vida, los que se estudiaron de forma independiente mediante análisis bivariado y regresión logística múltiple, encontrando que una menor calidad de vida global se relaciona con escolaridad baja, metástasis, presencia de síntomas clínicos de depresión y de ansiedad, lo que concuerda con los datos descritos previamente en la literatura científica; por un lado, la depresión está asociada de forma negativa con componentes de calidad de vida, como bienestar físico, social, emocional y funcional, y por otro lado es un predictor de una pobre calidad de vida relacionada con la salud (Kim et al., 2017), especialmente antes del tratamiento (Visser y Smets, 1998). Datos similares se han hallado en otros estudios respecto a la depresión y la ansiedad, las que aumentan al recibir el diagnóstico o el tratamiento, e incluso se mantienen altas en etapas avanzadas de la enfermedad. Tales aspectos emocionales se han visto sistemáticamente relacionados con una menor calidad de vida del paciente (López et al., 2017).

Una historia previa de depresión puede estar asociada a la privación hormonal, que confirma lo reportado en otras investigaciones respecto a la alta relevancia sobre la calidad de vida y el riesgo de esta población (Pirl, Siegel, Goode y Smith,
2002), ya que entre 10 y $40 \%$ experimenta problemas de salud mental, con depresión clínicamente significativa; además, aquellos que han recibido terapia hormonal tienen $23 \%$ más de probabilidades de desarrollar depresión, en comparación con aquellos que no la reciben, entre otros aspectos (Klaassen et al., 2017). Este grupo de pacientes presenta una prevalencia de depresión de 18.4\% después del tratamiento (Watts et al., 2014), que es 3-4\% mayor a la de hombres de edades similares de la población general (American Psychiatric Association, 2013).

Esta investigación confirma que el hecho de no tener pareja predice depresión, de acuerdo a Elliott, Scott, Monsour y Nuwayhid (2015). Lo anterior presenta considerables retos a la relación de pareja, por ejemplo en el aspecto íntimo; asimismo, los varones con un pobre ajuste de pareja tienen un mayor malestar emocional y experimentan más efectos adversos debidos al tratamiento. En cuanto a la presencia de ansiedad (Watts et al., 2014), la misma se relaciona con una menor calidad de vida, como se ha descrito en reportes previos respecto a los factores de riesgo que predisponen dicho estado emocional en pacientes con cáncer de próstata. En otros estudios, se incluyen factores médicos (sitio y curso clínico de la enfermedad, tipo de tratamiento, dolor, medicación), factores psicológicos (capacidad de afrontamiento, fase de desarrollo de la vida o trastornos previos del estado de ánimo) y factores sociales (disponibilidad de apoyo, presiones socioeconómicas y otros) (Mermelstein y Lesko, 1992).

Los hallazgos de este trabajo subrayan la gravedad de la depresión en pacientes con cáncer de próstata y sugiere la necesidad de un mejor enfoque clínico sobre los síntomas depresivos, específicamente porque hay recomendaciones recientes para identificar con mayor precisión la depresión entre estos hombres a fin de reducir su duración y severidad (Sharpley et al., 2017). Esto es relevante porque los pacientes con cáncer de próstata que padecen depresión están en mayor riesgo de cometer suicidio debido a su sexo, edad avanzada, diagnóstico de cáncer y a menudo su estado de viudez (Liebowitz, 1993; Massie, 1989; (Pirl et al., 2002).

En estudio previos se ha visto que los supervivientes del cáncer de próstata pueden experimen- 
tar una calidad de vida menor debido a ciertos síntomas urinarios, como incontinencia urinaria, molestias al orinar, trastornos del sueño, y dolor antes, durante y después del tratamiento (Bourke et al., 2015; Waller y Pattison, 2013), y que ello se asocia con una función sexual más pobre, en comparación con los efectos de la vigilancia activa, la radioterapia de haz externo y la braquiterapia (Broughman et al., 2017).

Desafortunadamente, la baja calidad de vida y los síntomas de depresión y ansiedad son problemas de funcionalidad y de salud mental que no se tratan porque no se diagnostican oportunamente en las etapas iniciales del cáncer de próstata, pues médicamente suelen considerarse como reacciones "normales", lo que puede derivar erróneamente en el manejo inadecuado de los síntomas observados al abordarlos de ese modo (Galindo et al., 2015), más que a la ocurrencia de dichos síntomas psicológicos por sí mismos y que acompañan la enfermedad. Este hecho puede explicar la razón de que la mayoría de los médicos oncólogos únicamente reconozcan la depresión en menos de la mitad de sus pacientes con depresión y remitan al psiquiatra especialista solo a $2 \%$ de los pacientes con cáncer y con problemas psiquiátricos severos (Passik et al., 1998).

Finalmente, se ha observado que la terapia cognitivo-conductual tiene efectos positivos en variables tales como la calidad de vida y los síntomas de ansiedad y depresión (Reynoso y Torres, 2018; Siddons, Wootten y Costello, 2013), por lo que se sugiere emprender investigaciones en esta área con el propósito de evaluar su efectividad en pacientes con cáncer, así como implementar políticas públicas para su difusión. El presente estudio es de corte exploratorio y tiene un tamaño de muestra pequeño, por lo que se requieren futuros trabajos para confirmar la relación de las diferentes variables estudiadas con los factores predictores observados y discutidos.
En conclusión, este estudio presenta datos originales que responden claramente al reciente reclamo de generar mayores conocimientos sobre la calidad de vida (Sierra et al., 2014; Vázquez et al., 2009) y la salud mental en pacientes con cáncer de próstata en la población mexicana. Los hallazgos indican que los síntomas clínicos de depresión y la ansiedad son predictores de una baja calidad de vida en este grupo de pacientes; que su prevalencia en todo el espectro de tratamiento es mayor que el de la población general, y que ciertos factores del apoyo social -como tener o no tener pareja, o ser pacientes jóvenes-se relacionan con la depresión y la ansiedad (Watts et al., 2014). Se considera también que, dados los resultados obtenidos, es necesario evaluar la calidad de vida, la ansiedad y la depresión de forma rutinaria en estos pacientes para identificar de manera temprana a aquellos que requieran apoyo psicológico para ofrecerles el tratamiento oportuno.

La evaluación de los factores psicológicos durante el proceso del cáncer de próstata, así como la oportuna canalización de los pacientes a los servicios de salud mental, pueden repercutir en un afrontamiento más adaptativo de la enfermedad, con lo que se asegura que la angustia psicológica no sea diagnosticada y tratada de un modo insuficiente o inconveniente (Galindo et al., 2014). A partir de la confirmación e identificación de factores explicativos de los síntomas depresivos y ansiosos en estos pacientes, fue posible identificar evidencias relevantes que permitirán poner en práctica intervenciones psicológicas dirigidas a la mejorar la calidad de vida y reducir o suprimir la depresión y la ansiedad en esta población clínica.

Tal conocimiento sugiere la necesidad de considerar dichas variables en los estudios psicológicos futuros, así como aumentar la cantidad de participantes, e incluir otros hospitales y otras variables biomédicas, con el objetivo de disponer de un modelo robusto para valorar la calidad de vida en este grupo de pacientes.

\section{REFERENCIAS}

Aaronson, N.K., Ahmedzai, S., Bergman, B., Bullinger, M., Cull, A., Duez, N.J., Takeda, F. (1993). The European Organization for Research and Treatment of Cancer, QLQ-C30: A quality-of-life instrument for use in international clinical trials in oncology. Journal of the National Cancer Institute, 85(5), 365-376. 
American Cancer Society (2016). Signos y síntomas del cáncer de próstata. Atlanta, GA: ACS.

American Psychiatric Association (2013). Diagnostic and Statistical Manual of Mental Disorders: DSM-5 (5th ed.). Washington, D.C: APA.

Bourke, L., Boorjian, S.A., Briganti, A., Klotz, L., Mucci, L., Resnick, M.J., Penson, D.F. (2015). Survivorship and improving quality of life in men with prostate cancer. European Urology, 68(3), 374-383.

Bray, F., Ferlay, J., Soerjomataram, I., Siegel, R.L., Torre, L.A. y Jemal, A. (2018). Global cancer statistics 2018: GLOBOCAN estimates of incidence and mortality worldwide for 36 cancers in 185 countries. CA: A Cancer Journal for Clinicians, 68(6), 394-424.

Broughman, J.R., Basak, R., Nielsen, M.E., Reeve, B.B., Usinger, D.S., Spearman, K.C., Chen, R.C. (2017). Prostate cancer patient characteristics associated with a strong preference to preserve sexual function and receipt of active surveillance. Journal of the National Cancer Institute, 110(4), 420-425. doi: 10.1093/jnci/djx218.

Cella, D. (1995). Measuring quality of life in palliative care. Seminars in Oncology, 22(2 Suppl. 3), 73-81.

Chen, R.C., Chang, P., Vetter, R.J., Lukka, H., Stokes, W.A., Sanda, M.G., Sandler, H.M. (2014). Recommended patient-reported core set of symptoms to measure in prostate cancer treatment trials. Journal of the National Cancer Institute, 106(7), 132-132.

Dale, W., Bilir, P., Han, M. y Meltzer, D. (2005). The role of anxiety in prostate carcinoma: a structured review of the literature. Cancer, 104(3), 467-478.

Elliott, K.E.J., Scott, J.L., Monsour, M. y Nuwayhid, F. (2015). Profiles of dyadic adjustment for advanced prostate cancer to inform couple-based intervention. Psychology \& Health, 30(11), 1259-1273.

Galindo, O., Benjet, C., Juárez, F., Rojas, E., Riveros, A., Aguilar, J.L., Servicio de Oncología Médica del Instituto Nacional de Cancerología (2015). Propiedades psicométricas de la Escala Hospitalaria de Ansiedad y Depresión (HADS) en una población de pacientes oncológicos mexicanos. Salud Mental, 38(4), 253-258.

Galindo, O., Muñoz, M., Campos, S., Rojas, E., Maya, M.C. y Alvarado, S. (2014). Tamizaje de aspectos psicooncológicos: validación de una lista de chequeo. Psicooncología, 10(2-3), 407-415.

Galindo, O., Rojas, E., Meneses, A., Aguilar, J., Álvarez, M.Á. y Alvarado, S. (2015). Propiedades psicométricas del Inventario de Ansiedad de Beck (BAI) en pacientes con cáncer. Psicooncología, 12(1), 51-58.

Jayadevappa, R., Malkowicz, S.B., Chhatre, S., Johnson, J.C. y Gallo, J.J. (2012). The burden of depression in prostate cancer: Depression and prostate cancer. Psycho-Oncology, 21(12), 1338-1345.

Kim, S.H., Seong, D.H., Yoon, S.M., Choi, Y.D., Choi, E. y Song, H. (2017). Predictors of health-related quality of life in Korean prostate cancer patients receiving androgen deprivation therapy. European Journal of Oncology Nursing, 30, 84-90.

Klaassen, Z., Arora, K., Wilson, S.N., King, S.A., Madi, R., Neal, D.E., Terris, M.K. (2017). Decreasing suicide risk among patients with prostate cancer: Implications for depression, erectile dysfunction, and suicidal ideation screening. Urologic Oncology, 6(2), 60-66. doi: 10.1016.

Liebowitz, M.R. (1993). Mixed anxiety and depression: should it be included in DSM-IV? The Journal of Clinical Psychiatry, 54(Suppl.4-7), 17-20.

López C., I., López F., L., Ríos G., E., Maisonobe, P., Hernández Y., E. y Sarmiento J., M. (2017). Impact of locally advanced or metastatic prostate cancer on the quality of life. Actas Urológicas Españolas, 41(6), 368-375.

Massie, M.J. (1989). Anxiety, panic, and phobias. En J. C. Holland y J. H. Rowland (Eds.): Handbook of Psychooncology: Psychological care of the patient with cancer (pp. 300-309). New York: Oxford University Press.

Mehnert, A., Lehmann, C., Graefen, M., Huland, H. y Koch, U. (2010). Depression, anxiety, post-traumatic stress disorder and health-related quality of life and its association with social support in ambulatory prostate cancer patients: Depression and anxiety in prostate cancer. European Journal of Cancer Care, 19(6), 736-745.

Mermelstein, H.T. y Lesko, L. (1992). Depression in patients with cancer. Psycho-Oncology, 1(4), 199-215.

Monga, U., Kerrigan, A.J., Thornby, J., Monga, T.N. y Zimmermann, K.P. (2005). Longitudinal study of quality of life in patients with localized prostate cancer undergoing radiotherapy. The Journal of Rehabilitation Research and Development, 42(3), 391-399.

Nead, K.T., Sinha, S., Yang, D.D. y Nguyen, P.L. (2017). Association of androgen deprivation therapy and depression in the treatment of prostate cancer: A systematic review and meta-analysis. Urologic Oncology, 35(11), 664.e1-664.e9. doi: 10.1016/j. urolonc.2017.07.016.

Nunnally, J.B. y Bernstein, J.I. (1995). Teoría psicométrica. México: McGraw-Hill.

Oñate O., L.F., Alcántara P., A., Vilar C., D., García H., G., Rojas C., E., Alvarado A., S. y Aiello C., V. (2009). Validation of the Mexican-Spanish version of the EORTC C30 and STO22 questionnaires for the evaluation of health-related quality of life in patients with gastric cancer. Annals of Surgical Oncology, 16(1), 88-95.

Oraá T., N., Rodríguez, J.A.C., Lentati, G.O., Pino, N.M., Sánchez F., M. y Martínez C., F.J. (2017). Efectos del tipo de tratamiento y grupo de riesgo en la calidad de vida y la información en pacientes con cáncer de próstata. Efectos del tipo de tratamiento y grupo de riesgo en la calidad de vida y la información en pacientes con cáncer de próstata. Psicooncología, 14(2-3), 241-254. 
Passik, S.D., Dugan, W., McDonald, M.V., Rosenfeld, B., Theobald, D.E. y Edgerton, S. (1998). Oncologists' recognition of depression in their patients with cancer. Journal of Clinical Oncology, 16(4), 1594-1600.

Pirl, W.F., Siegel, G.I., Goode, M.J. y Smith, M.R. (2002). Depression in men receiving androgen deprivation therapy for prostate cancer: a pilot study. Psycho-Oncology, 11(6), 518-523.

Reynoso N., N. y Torres D., J.A. (2018). Epidemiología del cáncer en México: carga global y proyecciones 2000-2020. Revista Latinoamericana de Medicina Conductual, 8(1), 9-15.

Sharpley, C.F., Christie, D.R.H., Bitsika, V., Agnew, L.L., Andronicos, N.M. y McMillan, M.E. (2017). Associations between reduced telomere length, depressed mood, anhedonia, and irritability in prostate cancer patients: Further evidence for the presence of "male depression"? Psycho-Oncology, 27(3),1072-1074. doi: 10.1002/pon.4547.

Siddons, H.M., Wootten, A.C. y Costello, A.J. (2013). A randomised, wait-list controlled trial: evaluation of a cognitive-behavioural group intervention on psycho-sexual adjustment for men with localised prostate cancer: Psycho-sexual adjustment to localised prostate cancer. Psycho-Oncology, 22(10), 2186-2192. doi: 10.1002/pon.3273.

Sierra K.L., Viveros, C., Martínez, G., Hernández, O. y Caballero, G. (2014). Calidad de vida en pacientes con cáncer de próstata, operados de prostatectomía radical laparoscópica. Revista Mexicana de Urología, 74(3), 133-140.

The WHOQOL Group (1998). The World Health Organization quality of life assessment (WHOQOL): development and general psychometric properties. Social Science \& Medicine, 46(12), 1569-1585.

Torre, L.A., Bray, F., Siegel, R.L., Ferlay, J., Lortet-Tieulent, J. y Jemal, A. (2015). Global cancer statistics, 2012. A Cancer Journal for Clinicians, 65(2), 87-108. doi: 10.3322/caac.21262.

Torres S., L.E., Espinoza G., R., Rojas M., R., Escamilla N., C., Vázquez S., R.A., Campuzano, J.C. y Lazcano P., E. (2016). Prostate cancer mortality according to marginalization status in Mexican states from 1980 to 2013. Salud Pública de Mexico, 58(2), 179-186.

Vázquez, L., Camarena, H., Martínez, J.Á. y Hernández, V. (2009). Estudio comparativo de calidad de vida en pacientes con cáncer de próstata con diferentes tratamientos iniciales. Revista Mexicana de Urología, 69(4), 143-146.

Visser, M.R. y Smets, E.M. (1998). Fatigue, depression and quality of life in cancer patients: how are they related? Supportive Care in Cancer, 6(2), 101-108.

Waller, J. y Pattison, N. (2013). Men's experiences of regaining urinary continence following robotic-assisted laparoscopic prostatectomy (RALP) for localised prostate cancer: a qualitative phenomenological study. Journal of Clinical Nursing, 22(3-4), 368-378.

Watts, S., Leydon, G., Birch, B., Prescott, P., Lai, L., Eardley, S. y Lewith, G. (2014). Depression and anxiety in prostate cancer: a systematic review and meta-analysis of prevalence rates. British Medical Journal Open, 4(3), e003901-e003901.

Wenzel, L. y Cella, D. (2005). Quality of life issues in gynecologic cancer. En W. J. Hoskins, C. A. Perez, R. C. Young et al. (Eds.): Principles and practices of gynecologic oncology (4th. ed.) (pp. 1333-1342). Philadelphia, PA: Lippincott, Williams \& Wilkins.

Zigmond, A.S. y Snaith, R.P. (1983). The Hospital Anxiety and Depression Scale. Acta Psychiatrica Scandinavica, 67(6), 361-370. 